\title{
A Comparative Study between Physical Properties of Compact and Ring Yarn Fabrics Produced from Medium and Coarser Yarn Counts
}

\author{
Ghada Ali Abou-Nassif \\ Fashion Design Department, Design and Art Faculty, King Abdul Aziz University, Jeddah, Saudi Arabia \\ Correspondence should be addressed to Ghada Ali Abou-Nassif; dr_ghada2013@yahoo.com
}

Received 8 August 2014; Revised 23 October 2014; Accepted 4 November 2014; Published 23 November 2014

Academic Editor: Arunangshu Mukhopadhyay

Copyright (c) 2014 Ghada Ali Abou-Nassif. This is an open access article distributed under the Creative Commons Attribution License, which permits unrestricted use, distribution, and reproduction in any medium, provided the original work is properly cited.

In compact spinning, fibers in drawing stage are condensed using air suction, which results in the produced yarns which significantly differ from ring spun yarns in their physical and mechanical properties. This study compares between physical properties of compact and ring yarn fabrics woven from coarser and medium yarn counts. The experimental results were statistically analyzed using ANOVA. The findings of this study revealed that woven fabrics produced from compact spun yarns are superior to those produced from ring spun yarns with respect to breaking strength, breaking elongation, abrasion resistance, tearing strength, and air permeability.

\section{Introduction}

Compact spinning, which is a modified version of ring spinning, produces a novel yarn structure, and the development of compact spinning has set new standards in yarn structure $[1,2]$. However, compact spinning is oriented to better fiber utilization and the high quality rather than higher productivity [3].

In conventional ring spinning, the zone between the nip line of the pair of delivery rollers and the twisted end of the yarn is called the "spinning triangle." This represents the critical weak spot of the ring spinning process. In this zone, the fiber assembly contains no twist. The edge fibers splay out from this zone and make little or no contribution to the yarn strength. Furthermore, the edge fibers lead to the familiar problem of yarn hairiness $[4,5]$.

In compact spinning, the fibers which have left the drafting system are guided via the perforated drums or lattice aprons over the openings of the suction slots. Following the air flow, the fibers move sideways and are consequently condensed. This condensing has such a favorable effect on the ratio of the width of the condensed fiber to yarn diameter that the spinning triangle is nearly eliminated. When spinning without a spinning triangle, almost all the fibers are incorporated into the yarn structure under the same tension. As the twist insertion takes place very close to the nip line, even short fibers can take up tension. This results in increased strength, as more fibers contribute to the yarn strength. The utilization degree of fibers can thus be increased [6-11].

According to previous studies, mechanical compact spinning significantly improves yarn tensile properties and reduces its hairiness $[12,13]$. Until now there are many studies about the comparison of the conventional ring and compact yarns properties [14-18].

Most researches operate on the comparison between compact and ring yarn woven fabrics focused on fabrics made from finer yarns. The objective of this study emphasizes the comparison between such fabrics woven from medium and coarser yarns.

\section{Experimental Work}

2.1. Material. Two sets of fabrics were woven from three weft yarn counts, namely, 20, 24, and $30 \mathrm{Ne}$. These weft yarns were spun from Egyptian cotton of Giza 86 (2.5\% span length, $30 \mathrm{~mm}$; fineness, 4.5 micronair; tenacity, $32.7 \mathrm{~g} / \mathrm{tex}$; 
and breaking elongation, 6\%). The weft yarn samples were spun on ring and compact spinning machines with twist factor 4 for all yarn counts. The yarn samples were spun on Reiters' ring frame using conventional and rotorcraft compact spinning mode (ROCOS). Experimental ring and compact spun yarns were separately used to produce plain woven fabrics on Air-jet weaving machine.

The Air-jet weaving machine has the following particulars:

(i) machine model: Toyoda 1998,

(ii) shedding mechanism: electronic dobby,

(iii) number of harness frames: 6 ,

(iv) machine running speed: $650 \mathrm{rpm}$,

(v) weave structure: plain 1/1,

(vi) fabric width: $160 \mathrm{~cm}$,

(vii) warp width: $165 \mathrm{~cm}$,

(viii) filling density: 60 picks/inch,

(ix) warp density: 66 ends/inch,

(x) weft yarn counts: 20,24, and 30/1 Ne,

(xi) warp yarn count: $24 / 1 \mathrm{Ne}$.

2.2. Laboratory Testing. Since the variation of the fabric samples was in the weft yarns and in spinning type, all tests were performed in the weft direction. Before testing, all fabric samples were conditioned for 24 hours under the standard atmospheric conditions $\left(20 \pm 2^{\circ} \mathrm{C}\right.$ temperatures, $65 \pm 2 \%$ relative humidity).

Breaking strength and elongation of all test specimens were measured on an Instron tensile strength tester model 4411 according to ASTM standard D 2256. The tests were carried out using $150 \mathrm{~mm}$ gauge length at an extension rate $300 \mathrm{~mm} / \mathrm{min}$. Twenty observations were taken for each fabric sample and then averaged. Air permeability tests were conducted on air permeability tester according to ASTM standard D737-96. Before testing, tension was applied on the fabric specimen to eliminate the wrinkle. Recorded air flow was divided by the exposed area of the test specimen. Exposed area of fabric samples was $10 \mathrm{~cm}^{2}$ under the pressure drop of 5 inch of water column. The average of twenty readings was taken for each woven fabric sample.

The abrasion resistances of the fabrics samples were tested on the Martindale pilling and abrasion tester at $9 \mathrm{kPa}$ pressure according to BS EN ISO 12947-2. A circular specimen $(38 \mathrm{~mm})$, mounted in a specimen holder and subjected to a defined load, is rubbed against an abrasive medium. The evaluation of the abrasion resistance is determined from the mass loss of specimen after specific amount (10,000 cycles) of rubs. Tearing strength of the woven fabric samples was determined by using Elmendorf tearing tester in accordance with ASTM standard test method D 1424. A template was used to cut fabric strips of $100 \pm 2 \mathrm{~mm}$ length and $63 \pm 0.15 \mathrm{~mm}$ width. The critical dimension, that is, the distance to be torn, was taken as $43 \pm 0.15 \mathrm{~mm}$ and the observation was recorded from the scale of the tester.

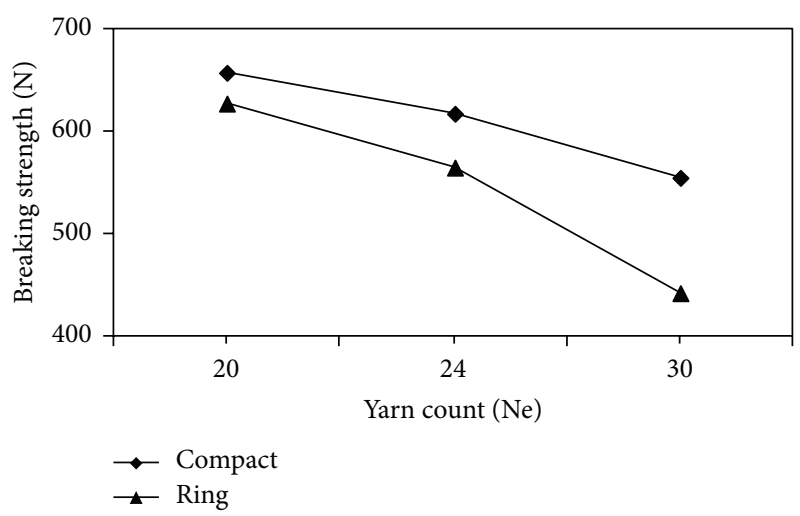

FIGURE 1: Effect of yarn counts and fabric type on the woven fabric breaking load.

\section{Results and Discussion}

3.1. Breaking Load. The breaking load test results are shown in Figure 1 and the statistical results of the breaking load property are given in Table 1 . The statistical analysis proved that spinning type and yarn count have a significant influence on the woven fabrics' breaking load at 0.01 significance level. It is shown that the weft yarn count was related inversely to the woven fabrics' breaking load. As the yarn count increases, the fabrics' breaking load decreases. The negative effect of weft yarn count on the breaking strength of the woven fabric may be due to the lower tensile strength of the constituent yarns with the increase in English count of the weft yarns.

The results also indicated that the fabrics produced with compact spun yarns have better tensile strength results compared to the fabrics produced with conventional ring spun yarns. The difference between the tensile strength of the compact and ring yarn fabrics is more pronounced in the case of finer yarns, that is, yarn count 30 Ne. However, in coarser yarns, compact spun yarns show a slight increase in tensile results compared to conventional ring spun yarns. The statistical analysis revealed that the average values of breaking load of compact yarn woven fabrics are 657,616 , and $554 \mathrm{cN}$ for weft yarns of counts 20,24 , and $30 \mathrm{Ne}$, respectively, whilst in the case of ring yarns woven fabrics the mean breaking load values of fabrics woven from weft yarn counts 20,24 , and $30 \mathrm{Ne}$ are 627,566, and $441 \mathrm{cN}$, respectively. The superior breaking strength of compact yarn fabrics over ring yarn ones may be ascribed to the exploitation of all fibers in the compact yarn cross-section which in turn increases the tensile properties of such yarns and then increases the breaking load of compact yarn fabrics.

3.2. Breaking Elongation. According to the statistical analysis' results listed in Table 2, the effect of weft yarn count and spinning type is significant on breaking elongation of woven fabrics under study at significance level 0.05 . The breaking elongation of ring and compact yarn fabrics against the weft yarn counts is plotted in Figure 2. As seen from this figure, the breaking elongation of ring and compact yarn fabrics is inversely related to the weft yarn counts. As the weft 
TABLE 1: Analysis of variance results for the effects of spinning type and yarn count on fabric breaking load.

\begin{tabular}{lccccc}
\hline Source of variation & SS & df & MS & $F$ & $P$ value \\
\hline Yarn count & 107.0126 & 2 & 53.50632 & 1433.845 & 0.000697 \\
Spinning type & 36.35882 & 1 & 36.35882 & 974.3318 & 0.001025 \\
Error & 0.074633 & 2 & 0.037317 & & 19.00003 \\
\hline Total & 143.4461 & 5 & & & \\
\hline
\end{tabular}

TABLE 2: Analysis of variance results for the effects of spinning type and yarn count on fabric breaking elongation.

\begin{tabular}{lcccccc}
\hline Source of variation & SS & df & MS & $F$ & $P$ value & $F$ crit \\
\hline Yarn count & 72.33333 & 2 & 36.16667 & 31 & 0.03125 & 0.039012 \\
Spinning type & 28.16667 & 1 & 28.16667 & 24.14286 & 18.51276 \\
Error & 2.333333 & 2 & 1.166667 & & \\
\hline Total & 102.8333 & 5 & & & \\
\hline
\end{tabular}

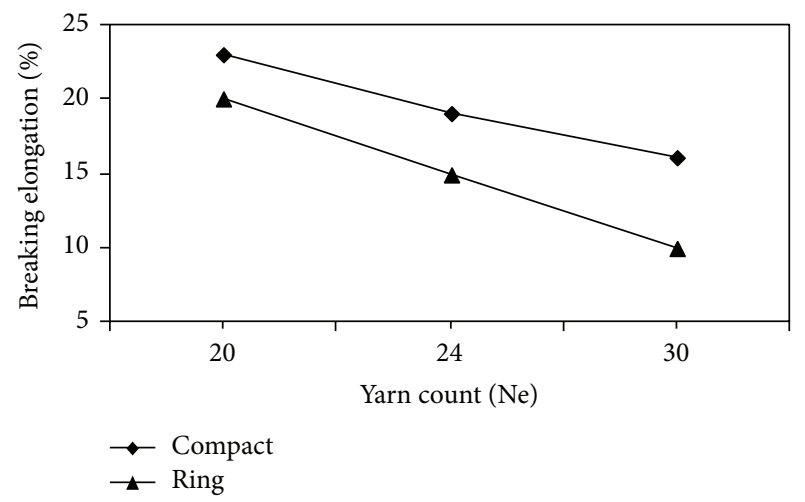

FigURE 2: Effect of yarn counts and fabric type on the woven fabric breaking elongation.

yarn counts increase the breaking elongation of both fabrics decreases. The inverse effect of yarn counts on fabric breaking elongation can be attributed to the decrease in yarn diameter with the increase of its count, which in turn leads to the reduction of breaking elongation of the constituent yarns, which reflected in lower fabric breaking elongation.

It is also observed that breaking elongation of compact yarn fabrics is higher than those produced from ring yarns. It is also apparent that the difference between breaking elongation of such fabrics is more pronounced at finer weft yarns. At coarser weft yarns the breaking elongation of compact yarn fabrics and that of ring yarn fabrics are close to each other. The statistical results revealed that the mean values of breaking elongation of compact and ring yarn fabrics are 23,19 , and $16 \%$ and 20,15 , and $10 \%$ for weft yarn counts 20,22 , and $24 \mathrm{Ne}$, respectively. The higher breaking elongation of compact yarn fabrics in comparison with ring yarn ones may be attributed to the higher elongation of compact yarns because of its exploitation of all fibers in the yarn cross section, which in turn leads to higher elongation of compact yarn fabrics.

3.3. Tearing Strength. Tearing strength is one of the important aspects of finished fabrics. It refers to the rupture of a fabric progressively along a line thread by thread. Tearing strength mainly depends on fiber, yarn, and fabric characteristics. The analysis of variance for the effects of weft yarn count and spinning type was listed in Table 3. From this table it is noticed that weft yarn count and spinning type have a significant influence on tearing strength of the woven fabrics at 0.05 significance level. The effect of weft yarn count on tearing strength of both compact and ring yarn fabric was shown in Figure 3. It is shown that weft yarn count has a negative effect on the tearing strength of both types of fabrics. As the weft yarn count increases the tearing strength decreases. It is also observed that the fabrics made from compact spun yarns are more tear resistant than those made of ring spun yarns. The tearing resistance of these fabrics is found to be higher in weft direction. The mean values of tearing strength of compact yarn fabrics are 4200,3900 , and $3600 \mathrm{gm}$ for weft yarn counts 20,24 , and $30 \mathrm{Ne}$, respectively. The average values of tearing strength of ring yarn fabrics were found to be 3900,3510 , and $3020 \mathrm{gm}$ for weft yarn counts 20,24 , and $30 \mathrm{Ne}$, respectively.

3.4. Abrasion Resistance. Abrasion is the mechanical deterioration of fabric components by rubbing them against another surface. Abrasion ultimately results in the loss of performance characteristics, such as strength, but it also affects the appearance of a fabric. The abrasion resistance of textile materials is affected by many factors (e.g., fiber fineness, yarn count, yarn type, and weave) in a very complex and as yet little understood manner.

In this study abrasion resistance of compact and ring yarn fabrics was characterized by the percent mass loss of the fabrics after 10000 rubs. As the mass loss increases the abrasion resistances decrease. The analysis of variance for the effects of spinning type and weft yarn count on the abrasion resistance of the woven fabrics under study was tabulated in Table 4. From this table it is noticed that spinning type and weft yarn count have a profound effect on fabric abrasion resistance at 0.05 significance level.

The effect of weft yarn count on percent mass loss of compact and ring yarn fabrics is illustrated at Figure 4. From this figure a decreasing trend is detected confirming 
TABLE 3: Analysis of variance results for the effects of spinning type and yarn count on fabric tearing strength.

\begin{tabular}{lccccc}
\hline Source of variation & SS & df & MS & $F$ & $P$ value \\
\hline Yarn count & 548433.3 & 2 & 274216.7 & 26.84013 & 0.035919 \\
Spinning type & 268816.7 & 1 & 268816.7 & 26.31158 & 0.035968 \\
Error & 20433.33 & 2 & 10216.67 & & 19.00003 \\
\hline Total & 837683.3 & 5 & & & 18.51276 \\
\hline
\end{tabular}

TABLE 4: Analysis of variance results for the effects of spinning type and yarn count on fabric tearing strength.

\begin{tabular}{|c|c|c|c|c|c|c|}
\hline Source of variation & SS & $\mathrm{df}$ & MS & $F$ & $P$ value & $F$ crit \\
\hline Yarn count & 39 & 2 & 19.5 & 39 & 0.025 & 19.00003 \\
\hline Spinning type & 13.5 & 1 & 13.5 & 27 & 0.035099 & 18.51276 \\
\hline Error & 1 & 2 & 0.5 & & & \\
\hline Total & 53.5 & 5 & & & & \\
\hline
\end{tabular}

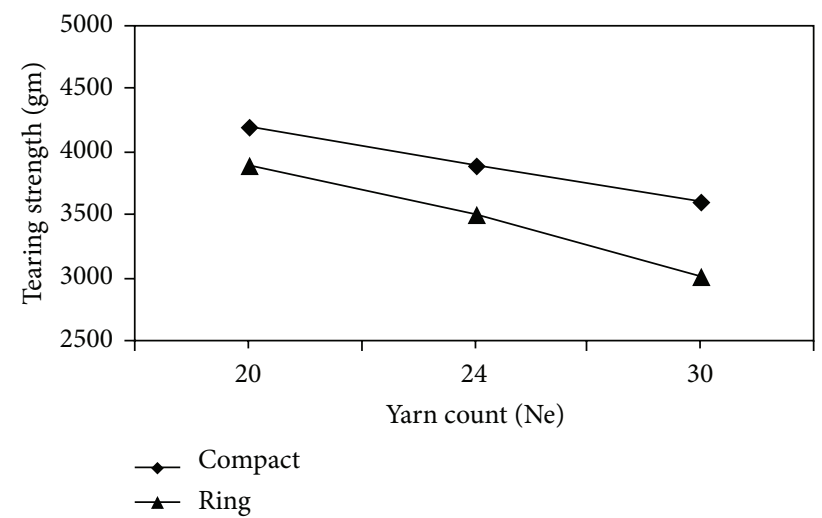

FIGURE 3: Effect of yarn counts and fabric type on the woven fabric tearing strength.

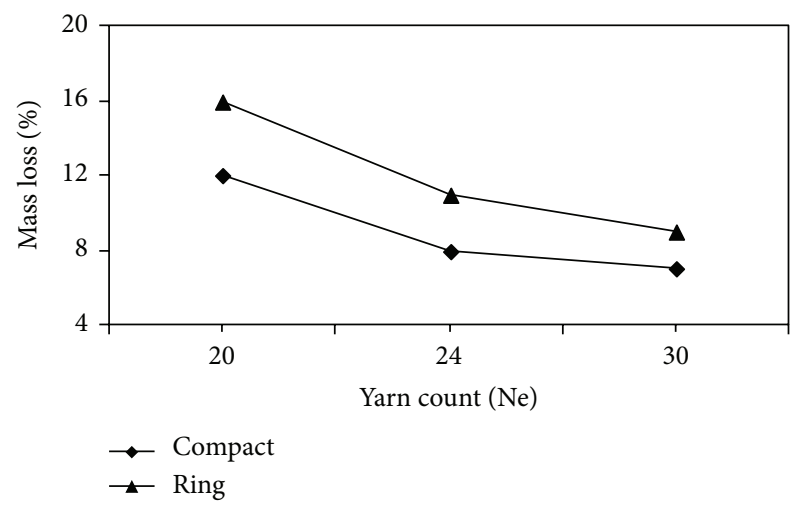

FIGURE 4: Effect of yarn counts and fabric type on the woven fabric abrasion resistance.

that as the weft yarn count increases the percent mass loss of the woven fabrics decreases. It was also clearly showed that woven fabrics made from compact yarn showed less mass loss after 10,000 cycles than the conventional ring based fabric. This means that compact yarn fabrics are more resistant to abrasion than those made from ring spun yarns. The statistical analysis proved that the percent mass loss of compact yarn fabrics made from yarn counts 20, 24, and $30 \mathrm{Ne}$ is $12 \%, 8 \%$, and $7 \%$, respectively, whereas in the case of ring yarn fabrics the percent mass loss was $16 \%, 11 \%$, and $9 \%$ for yarn counts 20,24 , and $30 \mathrm{Ne}$, respectively. Compact yarns based fabrics are expected to have lower friction values because of their uniform and less hairy structures and as a result higher abrasion resistance is expected. In contrast to the previous results, the difference between abrasion resistance of compact and ring yarn fabrics is more pronounced at coarser yarns.

3.5. Air Permeability. Air permeability is mainly affected by two parameters, porosity and fabric thickness. Since the fabric structure is the same for all fabric samples, plain weave, the fabric thickness will only affect the air permeability of the woven fabrics. Fabric thickness depends mainly on the yarn diameter, which in turn relies on yarn counts. Thus the only parameters which could affect the air permeability in this study are the yarn counts and yarn type.

The statistical analysis for the effects of spinning type and weft yarn count on the air permeability of the woven fabrics under study was tabulated in Table 5. From this table it is noticed that spinning type and weft yarn count have a significant influence on air permeability of such fabrics at 0.05 significance level.

The effects of weft yarn count on air permeability of compact and ring yarn fabrics are depicted in Figure 5. It can be seen from this figure that the relation between yarn count and air permeability of the woven fabrics under study is a direct relationship. As the filling yarn count increases the air permeability of compact and ring yarn fabrics increases. As the yarn count increases the fabric thickness decreases, which in turn leads to passage of much air through fabric cross section. It is also shown that woven fabrics made from compact spun yarns are more permeable than those made from ring spun yarns. This is because the compact spinning process helps for compacting yarn structure and thus leads to finer yarn cross section which in turn leads to more spaces between yarns in the fabric cross section.

The difference between the air permeability of compact and ring yarn fabrics is more pronounced in the case of 
TABLE 5: Analysis of variance results for the effects of spinning type and yarn count on fabric air permeability.

\begin{tabular}{lccccc}
\hline Source of variation & SS & df & MS & $F$ & $P$ value \\
\hline Yarn count & 330.3333 & 2 & 165.1667 & 39.64 & 0.024606 \\
Spinning type & 160.1667 & 1 & 160.1667 & 38.44 & 0.025042 \\
Error & 8.333333 & 2 & 4.166667 & & 19.00003 \\
\hline Total & 498.8333 & 5 & & & 18.51276 \\
\hline
\end{tabular}

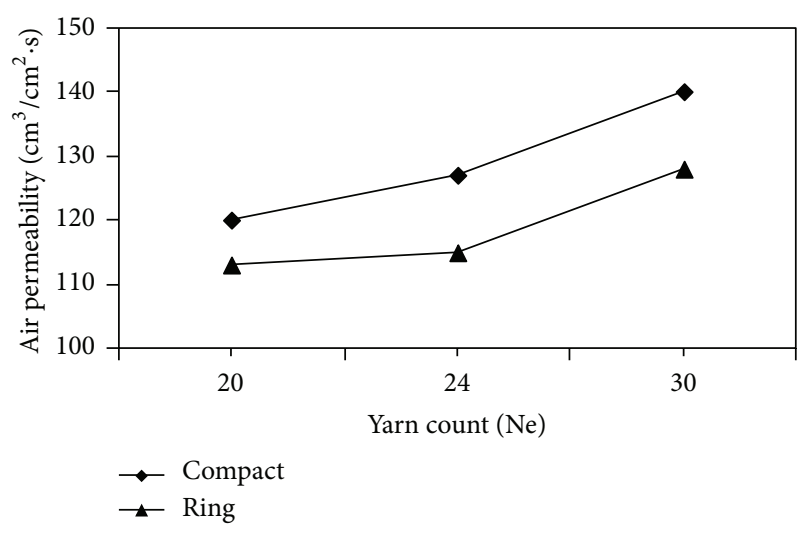

FIGURE 5: Effect of yarn counts and fabric type on the woven fabric air permeability.

medium yarn counts, while at coarser yarns, the air permeability of compact yarn fabrics and that of ring yarn fabrics are close to each other as shown from Figure 5. The statistical analysis proved that the average values for air permeability of compact yarn fabrics are 120,127, and $140\left(\mathrm{~cm}^{3} / \mathrm{cm}^{2} \cdot \mathrm{sec}\right)$ for weft yarn counts 20,24 , and $30 \mathrm{Ne}$, respectively. The corresponding air permeability values for ring yarn counts are 113,115 , and $128\left(\mathrm{~cm}^{3} / \mathrm{cm}^{2} \cdot \mathrm{sec}\right)$ for counts 20,24 , and $30 \mathrm{Ne}$, respectively.

\section{Conclusions}

Due to the difference in the yarn structure between compact and ring spun yarns, the physical and mechanical properties of fabrics woven from them will also be different. In this study physical and mechanical properties of fabrics woven from compact and ring spun yarns at coarser and medium counts were studied. The conclusion can be drawn as follows.

(i) There is a significant difference between the physical properties of compact and ring yarn fabrics in relation to breaking strength, breaking elongation, tearing strength, abrasion resistance, and air permeability.

(ii) It was found that compact yarn fabrics were superior to ring yarn fabrics in all these properties because of their compact and fineness compared to corresponding ring yarns.

(iii) The difference between compact and ring yarn fabrics was more pronounced at finer counts, whereas for coarser ones the properties of such fabrics are comparable approximately, and any one of the spinning systems can replace the other.

\section{Conflict of Interests}

The author declares that there is no conflict of interests regarding the publication of this paper.

\section{References}

[1] P. Artzt, "Short staple spinning on the way to new yarn structures and better raw material utilisation," International Textile Bulletin, vol. 45, no. 4, pp. 16-23, 1999.

[2] H. Stalder, "New spinning process ComforSpin," Melliand International, no. 6, pp. 22-25, 2000.

[3] U. Meyer, Compact Yarns: Innovation as a Sector Driving Force, Melliand International, 2000.

[4] G. Egbers, "The future of spinning and weaving," Melliand International, no. 3, pp. 34-36, 1999.

[5] W. Kampen, “The advantages of condensed spinning," Milliand International, no. 4, pp. 58-59, 2000.

[6] P. Artzt, "Possibilities of improving the efficiency of compact spinning," Melliand English, no. 3, pp. 19-21, 2002.

[7] K. P. S. Cheng and C. Yu, "A study of compact Spun Yarns," Textile Research Journal, vol. 73, no. 4, pp. 345-349, 2003.

[8] A. Hellwig, "Compact yarn in weaving - a new fashion or a must for high-quality fabrics," Melliand English, vol. 83, no. 6, pp. 8485, 2002.

[9] A. Olbrich, "The Air-Com-Tex 700 condenser ring spinning machine," Melliand International, vol. 6, pp. 26-29, 2000.

[10] J. Smekal, "Air-Com-Tex 700 for compact spinning yarns," Melliand International, vol. 7, no. 1, pp. 18-19, 2001.

[11] M. A. Saad and A. A. Almetwally, "Spinning techniques vs. yarn properties," Textile Asia, vol. 39, no. 7, pp. 35-41, 2008.

[12] Y. Beceren and B. U. Nergis, "Comparison of the effects of cotton yarns produced by new, modified and conventional spinning systems on yarn and knitted fabric performance," Textile Research Journal, vol. 78, no. 4, pp. 297-303, 2008.

[13] S. Ganesan, A. Venkatachalam, and V. Subramaniam, "Fibre migration in compact spun yarns: part II-mechanical compact yarn," Indian Journal of Fiber \& Textile Research, vol. 32, no. 2, pp. 169-172, 2007.

[14] J. R. Dash, S. M. Ishtiaque, and R. Alagirusamy, "Properties and processibility of compact yarns," Indian Journal of Fibre and Textile Research, vol. 27, no. 4, pp. 362-368, 2002.

[15] T. Jackowski, D. Cyniak, and J. Czekalski, "Compact cotton yarn," Fibres and Textiles in Eastern Europe, vol. 12, no. 4, pp. 22-26, 2004. 
[16] M. Nikolić, Z. Stjepanovič, F. Lesjak, and A. Štritof, "Compact spinning for improved quality of ring-spun yarns," Fibres and Textiles in Eastern Europe, vol. 11, no. 4, pp. 30-35, 2003.

[17] S. Mavruz and R. T. Ogulata, "Statistical investigation of properties of ring and compact yarns and knitted fabrics made of these kinds of yarns," Tekstil ve Konfeksiyon, vol. 18, no. 3, pp. 197-205, 2008.

[18] A. A. Almetwally and M. M. Salem, "Comparison between mechanical properties of fabrics woven from compact and ring spun yarns," Autex Research Journal, vol. 10, no. 1, pp. 35-40, 2010. 

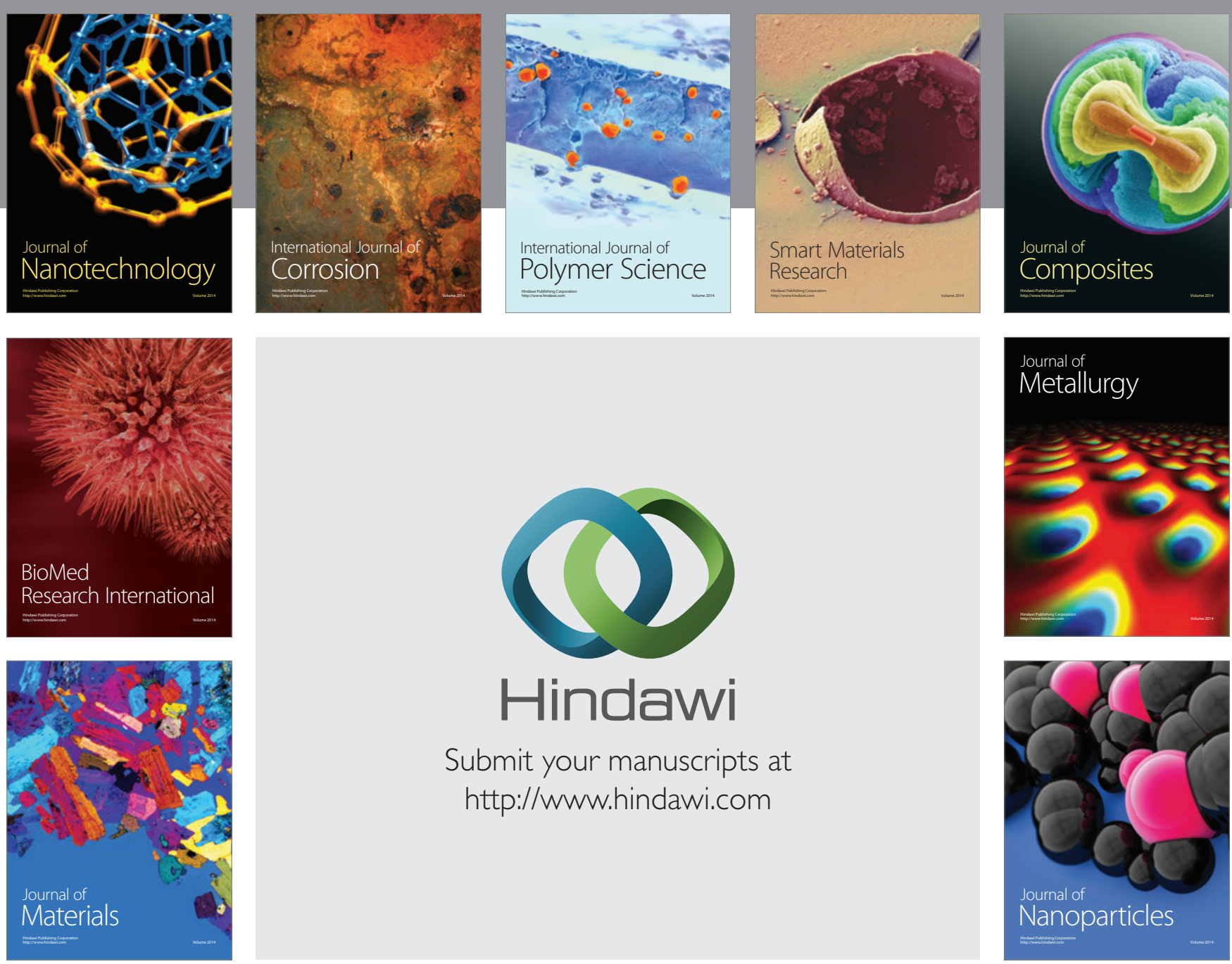

Submit your manuscripts at http://www.hindawi.com
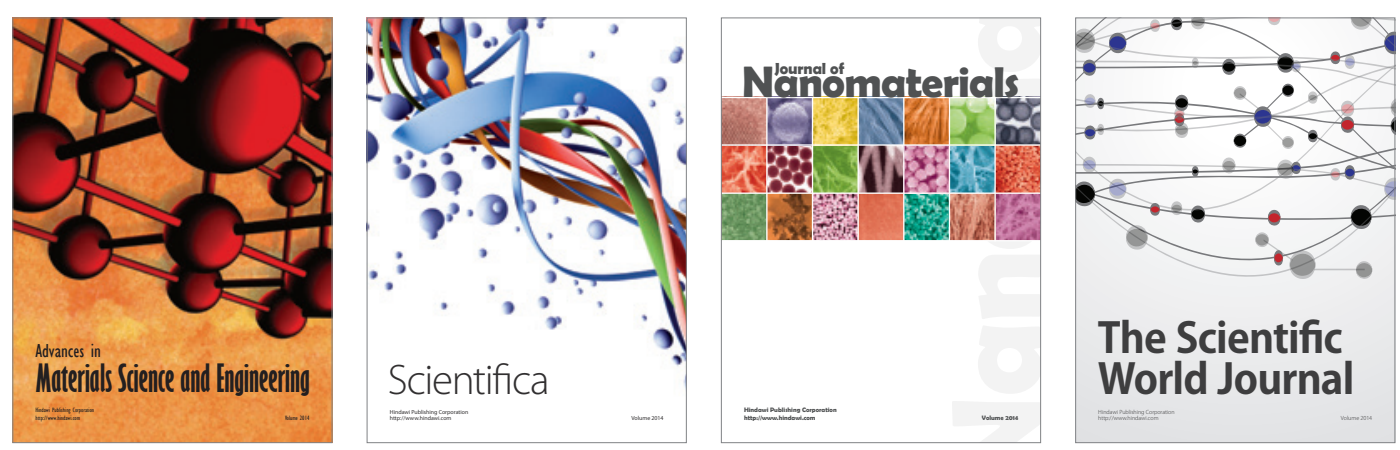

\section{The Scientific World Journal}
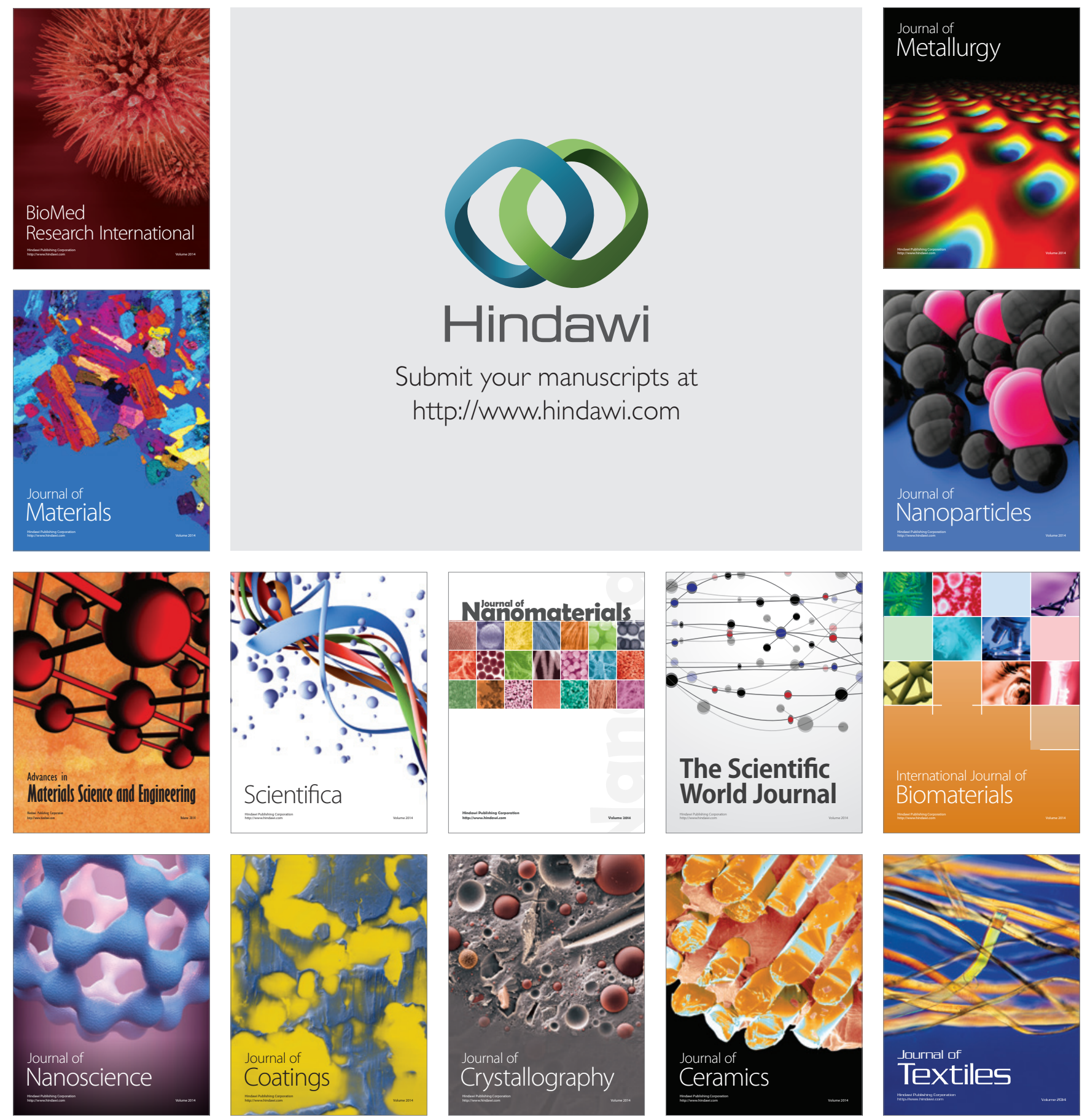